\title{
Interactive comment on "Dissolved Inorganic Nutrients in the Western Mediterranean Sea (2004-2017)" by Malek Belgacem et al.
}

\author{
Nico Lange \\ nmd.lange@gmail.com \\ Received and published: 19 November 2019
}

Interactive comment on "Dissolved Inorganic Nutrients in the Western Mediterranean Sea (2004-2017)" by Malek Belgacem et al.

This data-product is a great addition to the existing Mediterranean data products, filling gaps in time and space in respect to nutrient data in a "key" region. The paper itself is further a great presentation of this new data-product. Used methods are extensively outlined such that the end-user can easily understand the assigned adjustments and flags. Furthermore, the methods follow best-known practices as already used and verified in other recommended data-products (e.g. Crossover-analysis used in a.o. GLODAP). It is important to note that the original data are published as well, thus 
allowing later modifications/improvements of methods. Apart from one methodological issue concerning the calculated internal consistency no "big" content-related problems caught my attention. Finally, considering the understandable critics concerning the "incompleteness" of this data-product by not including new and existing MOOSE_GE cruises covering the central Golf of Lion, I would counteract that I see this product rather as a starting point of a living data product. I completely agree that in the next version the mentioned data should most definitely be included, but I would not deny the scientific value of this product. However, of course I have hopes for a living dataproduct being updated regularly as this is the only way to truly achieve a complete understanding of the nutrient dynamics in the med. Thanks to the authors, originators and everybody who has contributed!

Scientific Issues:

1) I have only detected one critical issue in this paper - the internal consistency calculation(s) and the resulting improvements. I am afraid that the weighted mean (WM) has been calculated wrongly (the used formula is correct!). One must use the "absolute" offsets in the WM-equation, i.e. an offset of 1.02 equals an "absolute" offset of $2 \%$, as it does for an offset of 0.98 . Thus in this example, the $2 \%$ in turn needs to be used in the WM-equation and not 1.02 or 0.98 . Otherwise, these might cancel each other out. This also implies that the lower the WM the better, e.g. a WM of $2 \%$ is better than a WM of $3 \%$ in terms of internal consistency. This WM reflects the absolute weighted mean offset of the data set compared to the reference data set, hence the smaller the WM the higher the internal consistency. Consequently, lines $316-318$ are misinterpretations of "wrong" numbers (crossover method application vs interpretation of the internal consistency)... The improvements should still be visible, however the calculations should be redone and the "correct" numbers should be given.

2) Concerning one minor issue - I am missing a presentation of the temporal distribution of the samples, i.e. the dates of the cruises. This would be nice to have to allow the detection of seasonal biases of the data-product (observational seasonal biases are

Printer-friendly version

Discussion paper
Interactive

comment

$\mathrm{C} 2$ 
hard to surpass and totally fine themselves but should be mentioned if present).

3) Lines 228 - 230 might be misleading: Weights are given according to the "confidence" in the determined offset of the compared profiles not necessarily the variance of the profiles themselves. I.e. the weighted mean offset of a given crossover-pair is weighted to the depth where the offsets of all compared profiles have the smallest variation. Usually this is the case in deep regions and yes, this indeed is strongly interlinked with the degree of variance of each profile... Please consider rephrasing.

Interactive comment on Earth Syst. Sci. Data Discuss., https://doi.org/10.5194/essd-2019-136, 2019. 